\title{
Twisting, an alternative strategy to compact granular materials
}

\author{
Karol Asencio ${ }^{1}$, Iker Zuriguel ${ }^{1}$, and Diego Maza ${ }^{1, \star}$ \\ ${ }^{1}$ Granular Media Lab. Departamento de Física y Matemática Aplicada. Universidad de Navarra. 31080, Pamplona, Navarra, \\ Spain.
}

\begin{abstract}
Nowadays, the common method to pack granular materials is to tap the ensemble against the gravity. Despite the apparent simplicity of that method, the asymptotic states reached by the tapped systems have strongly dependences on parameters like the shape of the tapping pulse, the container geometry or the ratio between lateral and axial dimensions. Beyond the restrictions imposed by the system boundaries, the particle shape (like rods or tetrahedrons) plays a central role in the evolution and the final state of the ensemble. In this work, we introduce an unconventional method for compacting granular ensembles by applying a sequence of alternating counterrotating pulses or "twists". By using spherical particles we analyze the efficiency of this method to achieve highly packed configurations.
\end{abstract}

\section{Introduction}

In almost the totality of granular storage processes, it is desirable to optimize the packing fraction in order to minimize the volume of the container. This, in principle, simple task implies to implement sophisticated technological solutions to drive the material to its optimal packing. The reason lies in the inherent complexity associated to the process of arrangement (or rearrangement) of any group of particles. From a fundamental point of view, the packing problem involves several challenges concerning: a) the existence of an well defined mean value of the volume fraction compatible with the history and boundary conditions of the packing; b) the mechanism that conduits any group of grains to this asymptotic value; and c) the dependence of this asymptotic value on the excitation parameter. Indeed, even in the case of monodisperse beads where a well defined maximum packing fraction has been recently predicted $[1,2]$, the geometry of the boundaries imposes important restrictions to this limit [3] implying that its calculation becomes a complex optimization problem [4]. Apart from the inherent difficulties of calculating this limit, the dynamical evolution to this magnitude and the possibility of approaching it asymptotically, are also relevant issues. Moreover, bead properties like friction and boundary conditions impose extra restrictions on the possibility of reaching such theoretical limit. Indeed, the states exhibited by an ensemble of particles that determine the typical average for the packing fraction, have relevant statistical features that have merited the introduction of novel theoretical frameworks to describe them [5].

\section{Experimental setup}

Nowadays, tapping is considered the canonical protocol employed to drive granular systems into statistically stationary equilibrium states, compatible with the boundary conditions of the system. In spite of the fact that this method is profusely used, its validity to bring the system to states statistically homogeneous is not completely clear in many circumstances and the results extracted from the experimental observations could appear contradictory. The most popular example of this is the differences between the "Chicago" and the "Rennes" experiences: both deal with the problem of packing grains by tapping but they are contained in cells with very different lateral dimension [6]. In the former case, a tall and narrow tube is filled with beads of a diameter one tenth smaller than the tube diameter; in the second, the particles' diameter was two orders of magnitude smaller than the diameter of the shallow cylindrical cell employed. In both setups, a large number of tapping pulses had to be applied to reach a stationary packing fraction: Moreover, the asymptotic volume fraction seems to have a monotonous dependence with the amplitude of the applied pulse. Nevertheless, the temporal evolution of the packing fraction with the number of taps is notably different: in the first case an inverse logarithmic growth seem to fit the results, whereas in the second, an stretched exponential was shown to be better to fit the temporal evolution. Moreover, significative differences exist in the figures obtained for the asymptotic packing fraction attained with the same tapping amplitude. Indeed, for tapping amplitude slightly larger than $\Gamma=3$ (where $\Gamma$ is the maximum acceleration applied to the setup normalized by the gravity), $\phi_{\max } \approx 0.635$ with the "Chicago" setup and $\phi_{\max } \approx 0.614$ with the "Rennes" one. The explanation given was that the strong confinement of the Chicago setup caused crys-

\footnotetext{
^e-mail: dmaza@unav.es
} 
tallization against the disorder induced by the convective regime developed when a large cell is used.

In this work we explore the possibility of obtaining highly packed arrangements when an large aspect ratio cell is used. In order to do that, we implement an alternative strategy to the tapping protocol which consists on twisting alternately a cylindrical container. Under this excitation, the granular sample self-organizes increasing its volume fraction by arranging the beads in superimposed layers that tends to reach its maximal packing fraction (see inset of Fig. 1). The temporal evolution of the volume fraction is analyzed for different twist amplitudes. Note that a similar strategy has been used previously but combined with tapping pulses [7]. The experimental setup consists of a cylindrical container of radius $R=87,0 \pm 0.1 \mathrm{~mm}$ made in transparent plexiglass with a base of aluminium (see Figure 1). In order to induce compaction the whole cylinder is rotated in alternating angular directions by a gearbox connected to a $1 / 2 \mathrm{HP}$ electrical motor which was controlled by an electronic variator. This allowed the variation of the angular velocity at will. The range of angular velocities goes from $2.3 \mathrm{~s}^{-1}$ to $17.8 \mathrm{~s}^{-1}$, which correspond to tangential velocities at the border of the cylinder $\mathrm{v}$, between $200 \mathrm{~mm} / \mathrm{s}$ and $1550 \mathrm{~mm} / \mathrm{s}$. This twisting produces an effective "shaking"; a perturbation that appears when the cell inverts its rotational direction. Just for comparison with other tapping experiments, we normalize the cell tangential acceleration, $\alpha=2 \mathrm{v} / \Delta t$ (where $\Delta t$ is the time necessary to invert the rotation) by the gravity acceleration: $\Gamma=\alpha / g$. The values of $\Gamma$ explored are in the range $0.3<\Gamma<1.01$, notably smaller than the used in other compaction experiments. Two type of beads are used, delrin spheres (radius $r=1.5 \mathrm{~mm}$ ) and PVC beads $(r=3 \mathrm{~mm})$. Delrin beads have a mass $m_{D}=0.018 \mathrm{~g}$ while PVC beads are one order of magnitude heavier, $m_{P}=0.200 \mathrm{~g}$ : the purpose of this was to compare the influence of inertial force on the final result. After the first

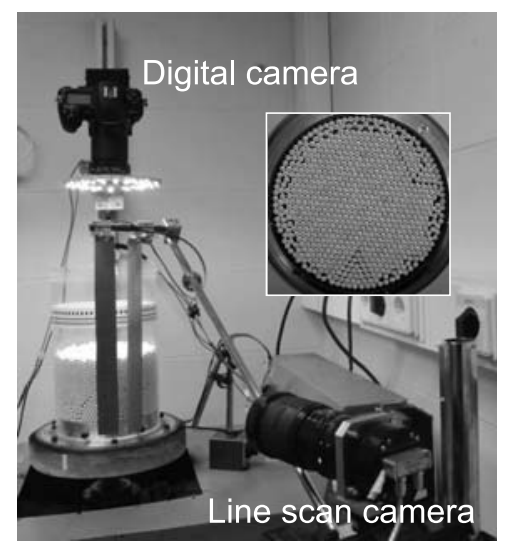

Figure 1. Experimental setup. An electrical motor rotates a cylindrical cell filled with spherical beds in alternating directions. The arrangements of the particles near the lateral surface could be obtained by using a line scan camera, and the free surface is registered by a digital camera. Inset: Upper view of a single layer of particles manually compacted near its maximum packing fraction. twist, the free surface of the material becomes almost flat independently of the configuration with which the grains were introduced in the cell. Therefore, the mean volumetric packing fraction, $\phi$ can be determined from the apparent volume of the material. That is, the packing fraction is calculated as the ratio between the volume of the beads poured in the cell and the volume of a cylinder of height equal to the mean value of the free surface. To determine this profile, a linear camera registered a lateral line of pixels of the cell rotating at small velocity. Then, the height of the packing is extracted from the profile of the free surface is obtained by image processing technics as is displayed in Fig. 2. Therefore, the relevant height fluctuations of the sample are due to some of the isolated beads resting on the top layer. Nevertheless, the maximum relative standard deviation observed in any typical experimental situation is below 5\%. Moreover, the free surface is only lightly deformed for the range of accelerations applied. Hence, although a systematic error exist in the estimation of the packing fraction due to the height overestimation, this error equally affects all the experimental results independently of the $\Gamma$ value.

Using a high speed camera we have also tested that, when the angular velocity is constant, in the frame of reference of the cylinder, particles do not move. Therefore, only when the angular velocity inverts its sign there is a stop-start motion that induces a shear stress within the material in the azimuthal direction. It is at this precise moment when particles move with respect to each other and therefore rearrangements occur. Moreover, the vertical load of the material also induces a torsional stress in the vertical direction; due to this, the free surface of the material suffers a net displacement respect to the base of the cell between successive twists.

\section{Experimental results}

Fig. 3 shows the packing fraction evolution of delrin particles as a function of the number of twists applied to the cell. The two extreme values of $\Gamma$ studied in this work have been presented. Like in similar experiments, we performed five experimental repetitions for each $\Gamma$ in order to check the repeatability of the results. Let us now discuss carefully each of these curves comparing the twisting results with previous, tapping ones. Clearly, the twisting protocol provides a very valuable tool to induce the compaction of the grain ensemble, primarily by using small amplitudes. Indeed, after $10^{4}$ twists the volume fraction obtained for $\Gamma=0.31$ is $\phi=0.66$ which is notably larger than the densest packing $(\phi<0.59)$ obtained for the smallest amplitude $(\Gamma=1.4)$ explored in the Chicago experiment [9]. The same argument could be extended to the results introduced by the "Rennes" group [8]. In this latter case, the final volume fraction is even lower for the smallest reported tapping amplitude. Note that these values of $\phi$ obtained for the smallest tapping intensities in that pioneering works $[8,9]$ were notably improved by performing an annealing procedure; that is, first tapping at high intensities and then reducing the intensity. In our 


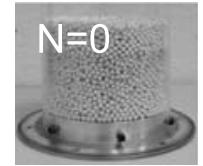

(a)

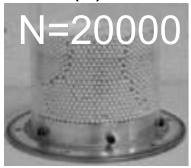

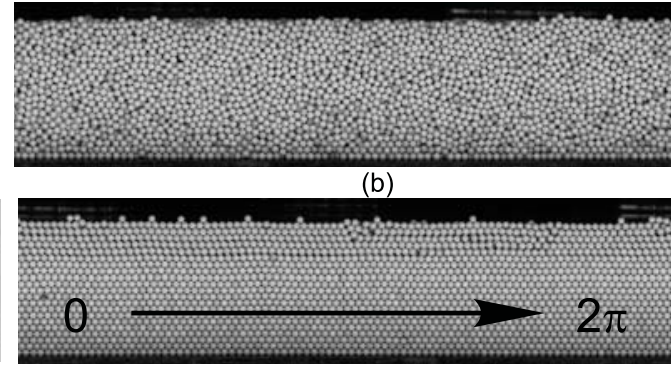

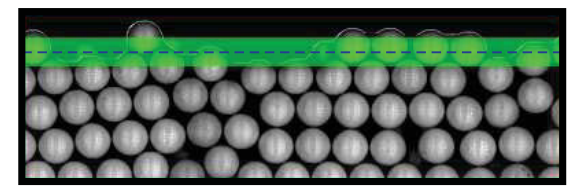

(c)

Figure 2. a) Pictures showing the initial (top) and final (down) states for a layer of $6 \mathrm{~mm}$ PVC beads submitted to 20000 twists. b) The image reconstructions obtained with a line scan camera showing all the particles in the external layer for these states. c) Detail of the top layer. Dashed line indicates the calculated mean height and the shadow region represent the interval defined by the standard deviation of the profile.

case, this annealing process seems to be not necessary to reach really well packed arrangements, although this should be deeply investigated in future works. Furthermore, preliminary results shown in Fig. 3 suggest that the packing fraction growth is logarithmic, until it reaches a cutoff value beyond which the packing fraction remains almost constant. For the case of $\Gamma=0.31$ only the logarithmic growth is readily visible as it seems that, after 30000 twists, the system has not reach the cutoff value. For the data corresponding to $\Gamma=1.01$, however, both regimes are clearly evidenced. When this large amplitude is applied, the volume fraction decreases during the first twists applied because some beads jump to the highest positions in the perimetrical layer of particles. Nevertheless, after a few twists, the packing fraction starts to increase as the sample arranges in a more compacted structure. At this stage, the volume fraction evolves between $\phi=0.6$ to $\phi=0.64$ in less than 500 twists. Clearly, this growth is markedly larger than the analogous in the tapping experiences using accelerations around the unity. After reaching the cutoff value, the packing slightly increases revealing important fluctuations which are related with the competition between domains as the ones shown in the right panel of Fig. 2. This observation suggests that the geometrical frustration induced by the ratio between the cylinder and particle sizes could be behind the fluctuations of the packing fraction around a well defined asymptotic mean value. As this ratio also determines the maximum volume fraction possible, in order to generalize the observed features, in Fig. 4 we compare the temporal evolution of packings of delrin $(r=1.5 \mathrm{~mm})$ and PVC beads $(r=3 \mathrm{~mm})$. Clearly, PVC beads present systematically lower volume fractions than delrin beads, although the global evolution of both type of particles is almost the same: a rapid growth until a cutoff value is reached. Beyond this point, the asymptotic volume fraction fluctuates around a mean value. Despite this similarity, their dynamics are not completely equal in both cases. Indeed, for larger spheres (PVC), the volume fraction fluctuations around the stationary state are more important than for the smaller ones (Delrin). Visual inspection of the external layer evidences the development of grain boundaries between ordered domains. Therefore, the fluctuations observed when the largest explored twist

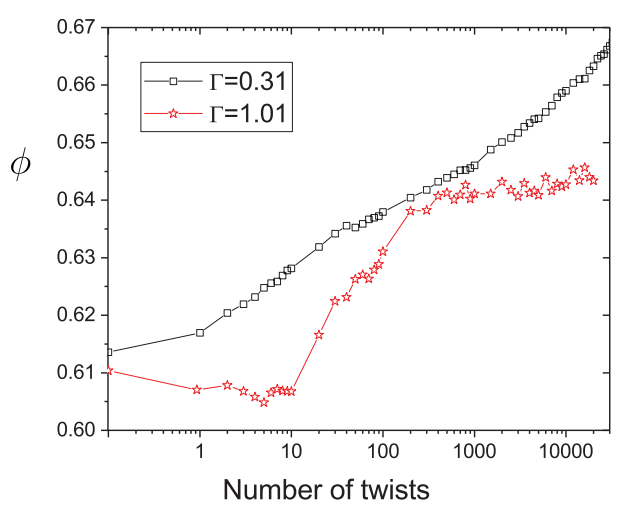

Figure 3. Volume fraction evolution of delrin particles as a function of the number of twists for the two limit accelerations studied: $\Gamma=0.31$ (square symbols) and $\Gamma=1.01$ (star symbols) as shown in the legend. Note the logarithmic scale in the $\mathrm{x}$ axis. Clearly, for low tapping amplitudes the volume fraction evolves quasi-monotonically, but for larger values of $\Gamma$ a different trend is observed: after a short interval of strong particle rearrangements (where the volume fraction even decreases) a rapid growth of the packing fraction is produced, followed by a (quasi) stationary state. Dotted lines are guides for the eyes.

amplitude is used seems to be related with the development of defect lines inside the ordered layers. This observation is compatible with the ideas introduced in $[14,15]$ where the microstructure of the arrangements is numerically analyzed in order to justify the evolution and fluctuations of the volume fraction. Importantly, we have also observed the sudden jumps reported in [15] and at a preliminary stage it is possible to associate these jumps to the competition between different orientational domains inside the system. This fact will be reported elsewhere.

Finally, let us note that the asymptotic limit of the volume fraction seems to reduce monotonically as the twist amplitude is increased in line with "Chicago" results. At this point, it should be stressed again that in $[8,10]$ this behavior was obtained only if an a annealing process was applied for the smallest intensities. In our case, we do not need such an annealing process as very high volume 


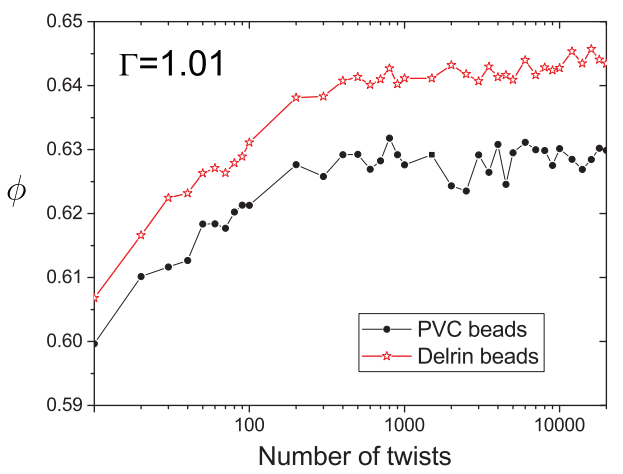

Figure 4. Volume fraction evolution for two different beads size: $5.95 \mathrm{~mm}$ diameter PVC beads (circle symbols) and $3 \mathrm{~mm}$ diameter delrin beads (star symbols). Note the logarithmic scale in the $\mathrm{x}$ axis. Despite the difference in the magnitude of the volume fraction, related with the difference in the diameter of the beads, the temporal evolution shows the same trends. Nevertheless, the strong fluctuations observed for large beads seem to be associated with the competition of ordered domains.

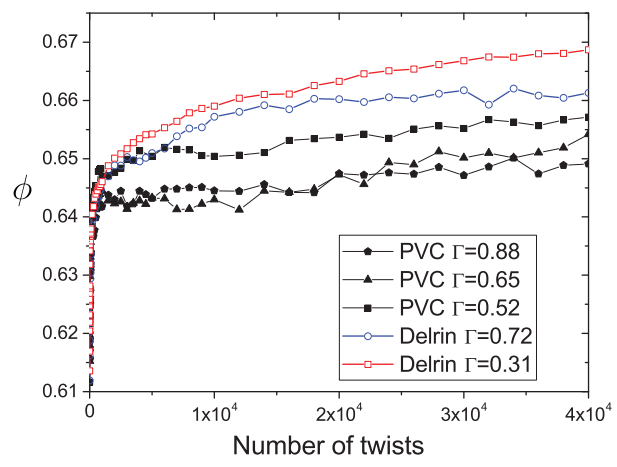

Figure 5. Volume fraction evolution for different twist amplitudes and the two types of beads used in this work as indicated in the legend. Clearly, independently of the mass of the beads, the asymptotic packing fraction follows a monotonous dependence with the magnitude of the perturbation applied: the stronger the intensity, the lower the final packing fraction. The highest volume fractions (obtained for the smaller intensities) have been achieved without applying any annealing protocol.

fractions could be reached if a sufficiently large number of twist cycles is applied as evidenced in Fig. 5.

\section{Conclusions}

In this work we have provided experimental evidence of the usefulness of applying alternating cycles of rotation (or "twists") to compact granular materials. Until now, the compaction of a granular ensembles has been mainly achieved by shaking the system which implies the application of sudden and large amplitude oscillations to small samples of material. Nevertheless, our protocol provides a simple mechanism to induce well ordered arrangements by shearing the material without need to provide external perturbation against the gravity force field (a condition needed in tapping). Indeed, the compaction of the system is only induced by the grains inertia during the reversal of the rotational motion of the container. Importantly, the observed asymptotic values of the packing fractions are larger than the ones previously reported in the literature. In addition, for low twist amplitudes the volume fraction seems to approach a limiting value compatible with the geometry of the boundary conditions, independently on the initial conditions and without applying any kind of annealing protocol [16]. Finally, let us mention that the comparison with the tapping results has been performed assuming the equivalence between twist and tap acceleration but this extreme will be confirmed using other parameters in line with the ideas introduced in $[11,12]$.

\section{Acknowledgments}

This work was funded by Ministerio de Economía y Competitividad (Spanish Government) through Projects No. FIS2011-26675 and FIS2014-57325. K. Asencio thanks Asociación de Amigos de la Universidad de Navarra for a scholarship.

\section{References}

[1] T. C. Hales, An overview of the Kepler conjecture. arXiv:math/9811071v2,(1998).

[2] Denis Weaire, Tomaso Aste, The Pursuit of Perfect Packing, 2nd Ed. (Taylor\& Francis, NY, London, 2008).

[3] See http://mathworld.wolfram.com/SpherePacking.html and references therein.

[4] http://hydra.nat.uni-magdeburg.de/packing/

[5] D. Bi,S. Henkes, K. Daniels, and B. Chakraborty Annu. Rev. Condens. Matter Phys. 6, 63 (2015).

[6] P. Richard, M. Nicodemi, R. Delannay, P. Ribière, and D. Bideau. Nature Materials 4, 121 (2005).

[7] K. Daniels, and R. Beheringer. Phys. Rev. Lett. 94, 168001 (2005).

[8] P. Philippe, and D. Bideau. Europhys. Lett. 60, 677 (2002).

[9] J.B. Knight, C. G. Fandrich, C.Ning Lau, H.M. Jaeger, and S. R. Nagel. Phys. Rev. E 51, 3957 (1995).

[10] E. Nowak, J. Knight, E. Ben-Naim, H. Jaeger, S. Nagel. Phys. Rev. E 57, 1971 (1998).

[11] G. Lumay, and N. Wandewalle. Phys. Rev. E 74, 021301 (2006).

[12] J.A. Dijksman, and M. van Hecke. Europhys. Lett. 88, 44001 (2009).

[13] E. Ben-Naim, J. Knight, E. Nowak, H. Jaeger, and S. Nagel. Physica D 123, 380 (1998).

[14] A. D. Rosato, and D. Tacoub. Powder Technology 109, 255 (2000).

[15] A. Rosato, O. Dybenko, D. Horntrop, V. Ratnaswamy, and L. Kondic. Phys. Rev. E 81, 061301 (2010).

[16] O. Caravente, and J.C. Ruiz-Suárez Phys. Rev. Lett. 95, 018001 (2005). 